\title{
A fast solution to the conjugacy problem in the four-strand braid group
}

\author{
Matthieu Calvez and Bert Wiest
}

Communicated by Pierre-Emmanuel Caprace

\begin{abstract}
We present an algorithm for solving the conjugacy search problem in the fourstrand braid group. The computational complexity is cubic with respect to the braid length.
\end{abstract}

\section{Introduction}

The conjugacy problem is one of the three famous decision problems in groups first formulated by Dehn in the early 20th century. The aim is to decide whether or not two given elements $x$ and $y$ of a group $G$ are conjugate in $G$, i.e. whether or not there exists an element $z$ in $G$ such that $x=z^{-1} y z$ (which we shall denote by $x=y^{z}$ ). If so, then an additional problem is to actually search for such a conjugating element $z$. These two problems are called CDP (the conjugacy decision problem) and CSP (the conjugacy search problem).

We know from Garside [17] that both CDP and CSP are solvable for the braid groups $B_{n}$, meaning that there exists an algorithm for solving these two problems in $B_{n}, n \geqslant 1$. In fact, the properties of braid groups discovered in [17] are now known to hold for a large class of groups, called Garside groups [13]; this class of groups contains, for instance, all Artin-Tits groups of spherical type [7].

A Garside group is a group equipped with a Garside structure, that is, roughly speaking, a lattice structure together with a distinguished element $\Delta$ satisfying some properties initially discovered by Garside for braids, in [17]. A crucial output of this is the left normal form for each element of a Garside group $G$ : this is a unique decomposition of the form $\Delta^{p} x_{1} \cdots x_{r}$ where the factors belong to the set of the so-called simple elements. This provides a measure of the length of an element: the canonical length $\ell(x)$ of an element $x \in G$ is the integer $r$ in the decomposition above. See Section 2 for more details. In the particular case of the braid groups, two distinct Garside structures are known. The classical one,

The first-named author was partially supported by a grant from Région Bretagne, by MTM201019355 and FEDER, and by FONDECYT through postdoctoral grant no. 3130569 and the Center of Dynamical Systems and Related Fields (project Anillo 1103). 
stemming from Garside's original article [17] and the dual one, introduced by Birman, Ko and Lee in [5].

Since Garside, several more, and more powerful, algorithms for solving CDP and CSP have been proposed $[15,18,19]$. We briefly recall that each of the algorithms $[15,18,19]$ for solving CDP and CSP in a Garside group $G$ is based on the calculation, for any given $x \in G$, of a finite non-empty subset $E_{x}$ of the conjugacy class of $x$, satisfying $E_{x}=E_{y}$ if and only if $x$ and $y$ are conjugate. The Super Summit Sets (SSS) [15], the Ultra Summit Sets (USS) [18] and the sets of Sliding Circuits (SC) [19] are three examples of such characteristic subsets. Unfortunately, despite the very high speed (in practice) of the most recent algorithms, the existence of a polynomial bound on the algorithmic complexity (with respect to the length of the input) is still an open problem, even in the case of the braid groups.

The main result of the current article is the following:

Theorem 1.1. There exists an algorithm which solves CDP and CSP in the braid group $B_{4}$ and whose algorithmic complexity is cubic with respect to the length of the input braid words.

We are not able to prove Theorem 1.1 using only the tools of Garside theory. We shall also use a geometric point of view on braids. It is known (see e.g. [1]) that the braid group $B_{n}(n \geqslant 1)$ can be identified with the mapping class group of the $n$-times closed punctured disk $\mathbb{D}_{n}$. In this context, braids can be classified according to their dynamical properties, in the following trichotomy (the NielsenThurston classification) $[11,16]$ : a braid $x$ is

- periodic if there exists an integer $m$ such that $x^{m} \in Z B_{n}=\left\langle\Delta^{2}\right\rangle$,

- reducible if there exists a non-empty family $\mathcal{F}$ (called the canonical reduction system) of isotopy classes of non-degenerate disjoint simple closed curves in $\mathbb{D}_{n}$ (non-degenerate means not null-homotopic, not homotopic into a puncture and not boundary-parallel) such that

- the $x$-action leaves $\mathcal{F}$ invariant,

- no element of $\mathcal{F}$ intersects another isotopy class of simple closed curves in $\mathbb{D}_{n}$ that is invariant under some power of $x$,

- pseudo-Anosov (pA) otherwise.

We remark that the definition of "reducible" most frequently found in the literature also encompasses certain periodic elements. In this paper we only apply the word "reducible" to the braids which would usually be called "reducible non-periodic". 
The paper [2] proposes a program, based both on the above classification and on Garside theory, for solving CDP and CSP in the braid groups in polynomial time with respect to both the length of the input braid words and their number of strands. A first step in this program is the construction of a polynomial time algorithm for deciding the dynamical type of any given braid ([2, Open Question 1]).

In [9], the authors answered this question in the case of the group $B_{4}$ : they produced an algorithm of complexity $O\left(\ell^{2}\right)$ to decide the Nielsen-Thurston type of any given four-strand braid of length $\ell$. Thus in the group $B_{4}$, in order to solve CDP and CSP it is sufficient to solve these problems for pairs of elements which are known to be of the same dynamical type (as pairs of braids of different dynamical type are never conjugate).

The algorithm given in [9] also implies a solution to CDP and CSP for reducible four-strand braids of length at most $\ell$ in time $O\left(\ell^{2}\right)$. The main lemma here is that for braids with at most 3 strands and of length at most $\ell$, the problems CDP and CSP are solvable in time $O\left(\ell^{2}\right)$, see [9].

The case of periodic braids is treated in [4], where an algorithm of complexity $O\left(\ell^{3} n^{2} \log n\right)$ for solving CDP and CSP for periodic braids with $n$ strands of canonical length at most $\ell$ is presented.

In order to prove Theorem 1.1, we thus have to produce an algorithm of complexity at most $O\left(\ell^{3}\right)$ capable of solving CDP and CSP for pseudo-Anosov fourstrand braids of length at most $\ell$. Technically, our main contribution is the following result, which gives a partial affirmative answer (in the special case of pseudoAnosov four-strand braids) to [2, Open Question 2]: using the vocabulary of [19], if a four-strand pseudo-Anosov braid is rigid (meaning, roughly speaking, that the normal form is as simple as possible), then its set of Sliding Circuits (and also its Ultra Summit Set) is "small":

Theorem 1.2. For every braid $x$ in $B_{4}$ which is pseudo-Anosov and rigid with respect to the dual Garside structure, the cardinality of $\mathrm{SC}(x)$ for the dual structure is bounded above by $O\left(\ell(x)^{2}\right)$.

This result implies that the algorithm given in [20] for solving CDP and CSP has complexity $O\left(\ell^{3}\right)$ when applied to two four-strand braids which are of length at most $\ell$, pseudo-Anosov and rigid in the dual structure.

The rest of the proof of Theorem 1.1 thus has to consist of a reduction to the rigid case. We prove, thanks mostly to Masur-Minsky's conjugacy bound [23]:

Theorem 1.3. There is an algorithm with the following properties:

- as input, it takes two pseudo-Anosov braids $x, y \in B_{n}$ of canonical length at most $\ell$, 
- as output, it yields an integer $s$ and $n$-strand braids $\bar{x}, \bar{y}, z_{1}$ and $z_{2}$ such that $\bar{x}$ and $\bar{y}$ are rigid and satisfy $\bar{x}=\left(x^{s}\right)^{z_{1}}$ and $\bar{y}=\left(y^{s}\right)^{z_{2}}$,

- for any fixed $n$, the complexity is $O\left(\ell^{2}\right)$.

Then, for $n=4$, the quick solution to CDP/CSP for two rigid pseudo-Anosov braids $\bar{x}$ and $\bar{y}$ results in a quick solution to the same problems for the initial pseudo-Anosov braids $x$ and $y$, because of the uniqueness of roots of pseudoAnosov braids [21]. We are now in a position to describe the algorithm promised by Theorem 1.1:

Algorithm. INPUT: $x$ and $y$ two elements of the four-strand braid group.

OUTPUT: whether or not $x$ and $y$ are conjugate and, if they are, an element $z \in B_{4}$ so that $x=y^{z}$.

(1) Determine the dynamical types of $x$ and $y$, using [9]. If they are not the same, answer " $x$ and $y$ are not conjugate" and STOP.

(2) If $x$ and $y$ are periodic, use [4] and STOP.

(3) If $x$ and $y$ are reducible, use [9] and STOP.

(4) If $x$ and $y$ are pseudo-Anosov, use the algorithm of Theorem 1.3 in order to produce $s, \bar{x}, \bar{y}, z_{1}, z_{2}$ with the required properties.

(5) Apply [20, Algorithm 3] to $\bar{x}$ and $\bar{y}$. If $\bar{x}$ and $\bar{y}$ are conjugate, then this algorithm produces $c \in B_{4}$ such that $\bar{x}=\bar{y}^{c}$. In this case answer " $x$ is conjugate to $y$ by $z_{2} c z_{1}^{-1}$ " and STOP.

(6) Answer " $x$ and $y$ are not conjugate."

Remark 1.4. Note that the preceding algorithm is completely explicit (in particular, we shall prove Theorem 1.3 by explicitly constructing the required algorithm). However, our cubic bound on the algorithmic complexity in Theorem 1.1 is not explicit, and this is due to the non-explicitness of Masur-Minsky's conjugacy bound [23]. More precisely, there are two things for which we have only existence proofs, not explicit constructions, namely

(i) the quadratic function bounding the size of $\mathrm{SC}(x)$ in Theorem 1.2 (see Proposition 5.12),

(ii) the linear function bounding the number of cyclic slidings in the proof of Theorem 1.3, and thus the quadratic bound on the complexity of the algorithm in Theorem 1.3.

Remark 1.5. The proposed algorithm itself was probably known to experts in the field. As is explained above, Steps (1) to (3) were known to have polynomial 
complexity; thus our main contribution is showing the polynomial complexity of Step (5) (Theorem 1.2) and Step (4) (Theorem 1.3, resting on Masur-Minsky's linear bound).

This paper is organized as follows. In Section 2 we recall some definitions and basic facts about Garside groups. Assuming Theorem 1.2, we prove Theorem 1.3 and Theorem 1.1 in Section 3. The two last sections are devoted to the proof of Theorem 1.2.

After this paper was completed, Sang Jin Lee communicated to us that a (degree 4) polynomial solution to the conjugacy problem in the four-strand braid groups was already present in his unpublished PhD thesis [22]. He notably showed a (degree 4) polynomial bound as in Theorem 1.2, also in the context of the dual Garside structure.

\section{The conjugacy problem in Garside groups}

In this section we recall the definition and some general facts concerning Garside groups and the known solutions to the conjugacy problem.

Definition 2.1 ([19]). Let $G$ be a group. We say that $G$ is a Garside group (of finite type) if it admits a submonoid $P$ satisfying $P \cap P^{-1}=\{1\}$ (the monoid of positive elements) and a distinguished element $\Delta \in P$ (Garside element) such that the following properties hold:

(1) The partial order $\preccurlyeq$ on $G$ defined by $x \preccurlyeq y$ if and only if $x^{-1} y \in P$ is a lattice order, meaning that any two elements admit a greatest common divisor and a least common multiple (note that it is invariant under left-multiplication); this is called the prefix order.

(2) The set $\{x \in G, 1 \preccurlyeq z \preccurlyeq \Delta\}$ is finite and generates $G$; its elements are called simple elements.

(3) Conjugation by $\Delta$ preserves the submonoid $P$.

(4) For every $x \in P-\{1\}$,

$$
\|x\|=\sup \left\{k: \exists a_{1}, \ldots, a_{k} \in P-\{1\}, x=a_{1} \cdots a_{k}\right\}<\infty .
$$

In this context we also say that the triple $(G, P, \Delta)$ is a Garside structure of finite type for $G$.

Throughout this section, $G$ denotes a Garside group with Garside structure $(G, P, \Delta)$. The greatest common divisor of two elements $x, y$ of $G$ with respect to $\preccurlyeq$ is denoted by $x \wedge y$. We denote by $\tau$ the inner automorphism associated to $\Delta$. 
Because it preserves $P$ and hence $\preccurlyeq$, the automorphism $\tau$ induces a permutation of the (finite) set of simple elements, and since these elements generate $G, \tau$ is of finite order.

We also recall that every simple element $s$ possesses a right complement $\partial(s)$ defined by the formula

$$
\partial(s)=s^{-1} \Delta .
$$

This allows us to define the notion of left-weightedness: a pair of simple elements $s_{1}, s_{2}$ is said to be left-weighted if

$$
\partial\left(s_{1}\right) \wedge s_{2}=1
$$

or, in other words, if $s_{1}$ is the greatest simple divisor of $s_{1} s_{2}$.

A crucial property of Garside groups is the existence of unique (left) normal forms:

Proposition $2.2([13,15])$. Let $x \in G$. There exists a unique decomposition

$$
x=\Delta^{p} x_{1} \cdots x_{r},
$$

where $r$ is a non-negative integer, the factors $x_{i}$ are simple elements such that $x_{1} \neq \Delta, x_{r} \neq 1$ and (if $r \geqslant 2$ ) the pair $x_{i} x_{i+1}$ is left-weighted for $i=1, \ldots, r-1$.

If the left normal form of $x$ is $\Delta^{p} x_{1} \ldots x_{r}$, the integers $p$ and $r$ are called the infimum and the canonical length of $x$, and they are denoted by $\inf (x)$ and $\ell(x)$. They are, respectively, the maximal integer $p \in \mathbb{Z}$ such that $\Delta^{p}$ is a prefix of $x$, and the minimal number of simple factors needed to express the element $\Delta^{-p} x$. The supremum $\sup (x)$ is the quantity $p+r$. For every element $x$ of $G$, one has $\sup (x)=\min \left\{k \in \mathbb{Z}: x \preccurlyeq \Delta^{k}\right\}$.

We observe that the elements of canonical length zero are precisely the powers of $\Delta$; these elements are as simple as possible within their conjugacy class. For an element $x$ of positive canonical length and normal form $\Delta^{p} x_{1} \cdots x_{r}$, one can define its initial and final factor as

$$
\iota(x)=\tau^{-p}\left(x_{1}\right), \quad \varphi(x)=x_{r} .
$$

Remark 2.3. The set of simple elements, taken as a generating set of $G$, induces a length function on $G$ : the length $|x|$ of an element $x$ of $G$ is, by definition, the smallest possible length of a word representing $x$ whose letters are simple elements or their inverses. We note that we always have $\ell(x) \leqslant|x|$. We also have the following relations, for any $x$ satisfying $\inf (x)=p$ and $\ell(x)=r$ (see [12,13]):

$$
|x|= \begin{cases}p+r & \text { if } p \geqslant 0, \\ r & \text { if } p<0 \text { and }|p| \leqslant r, \\ |p| & \text { if } p<0 \text { and }|p|>r .\end{cases}
$$


We recall that $G$ is equipped with different operations which are defined in terms of normal forms, each corresponding to a particular conjugation.

Definition 2.4 ([15]). Let $x \in G$ with normal form $x=\Delta^{p} x_{1} \cdots x_{r}$. Suppose $r \geqslant 1$. We define

- the cycling $\mathbf{c}(x)=x^{\iota(x)}=\Delta^{p} x_{2} \cdots x_{r} \tau^{-p}\left(x_{1}\right)$,

- the decycling $\mathbf{d}(x)=x^{\varphi(x)^{-1}}=\Delta^{p} \tau^{p}\left(x_{r}\right) x_{1} \cdots x_{r-1}$.

If $\ell(x)=0$, we also define $\mathbf{c}(x)=\mathbf{d}(x)=x$.

More recently, Gebhardt and González-Meneses introduced a new type of conjugation which combines cycling and decycling into a single, conceptually simpler, operation:

Definition 2.5 ([19]). Let $x \in G$ with normal form $x=\Delta^{p} x_{1} \cdots x_{r}$. Suppose $r \geqslant 1$. We define the preferred prefix of $x$ by the formula

$$
\mathfrak{p}(x)=\iota(x) \wedge \partial(\varphi(x)) .
$$

Cyclic sliding is the operation $\mathfrak{s}$ defined by

$$
\mathfrak{s}(x)=x^{\mathfrak{p}(x)} .
$$

If $\ell(x)=0$, then we also define $s(x)=x$.

The aim of cycling, decycling and cyclic sliding is to simplify the left normal forms; note that we have $\ell(\mathbf{c}(x)) \leqslant \ell(x), \ell(\mathbf{d}(x)) \leqslant \ell(x)$ and $\ell(\mathfrak{s}(x)) \leqslant \ell(x)$. Also note that all of the three operations commute with the automorphism $\tau$. Finally we make the following computational observation, which will be very useful in the sequel: if $x$ is an element of $G$ given in normal form, then its respective results under the three previously defined special conjugations can be computed in time $O(\ell(x))$.

Proposition 2.6 ([15]). Let $x \in G$.

(i) The subset of the conjugacy class of $x$ consisting of all elements with minimal canonical length is finite and non-empty. Its elements have simultaneously maximal infimum and minimal supremum. This subset is called the Super Summit Set of $x$, and denoted by $\operatorname{SSS}(x)$.

(ii) There exist $k_{0}, l_{0} \in \mathbb{N}$ such that $\mathbf{c}^{k}\left(\mathbf{d}^{l}(x)\right) \in \operatorname{SSS}(x)$ for every $k \geqslant k_{0}$ and $l \geqslant l_{0}$.

(iii) There exists $k_{0} \in \mathbb{N}$ such that $\Im^{k}(x) \in \operatorname{SSS}(x)$ for every $k \geqslant k_{0}$ (see [19]). 
The observation that $\mathfrak{s}$ preserves $\operatorname{SSS}(x)$ implies that the set of periodic points of $\mathfrak{s}$ in the conjugacy class of $x$ is a (finite) non-empty subset of $\operatorname{SSS}(x)$; this defines another conjugacy invariant.

Definition 2.7 ([19]). Let $x \in G$. The set of Sliding Circuits of $x$ is the set of all conjugates of $x$ which are periodic points of the cyclic sliding operation. That is, $\operatorname{SC}(x)=\left\{y \in x^{G}: \exists k \in \mathbb{N}, \mathfrak{s}^{k}(y)=y\right\}$.

An important example of fixed points of $s$ are the so-called rigid elements:

Definition 2.8. Let $x \in G$ with normal form $x=\Delta^{p} x_{1} \cdots x_{r}$. Suppose $r \geqslant 1$. We say $x$ is rigid if the pair $\varphi(x) \iota(x)$ is left-weighted.

In particular, an element of canonical length 0 is not rigid. Let us mention that ([2, Lemma 3.5]) an element $x \in G$ is rigid if and only if $x^{-1}$ is.

One very useful quality of the Super Summit Set, which is not known to hold for the set of Sliding Circuits (see Proposition 3.2), is that it can quickly be reached by iterated cyclic sliding:

Theorem 2.9 ([6,19]). Let $G$ be a Garside group. Then there exists a constant $\alpha$, which depends only on the group $G$ and its Garside structure, such that for every $x \in G, \mathfrak{s}^{\ell(x) \cdot \alpha}(x) \in \operatorname{SSS}(x)$.

For instance, in the case of the classical Garside structure on $B_{n}$, we have $\alpha=\frac{n(n-1)}{2}$, and $\alpha=n-1$ for the dual structure. This result has a very important algorithmic application: it yields an algorithm of complexity $O\left(\ell^{2}\right)$ for calculating an element $y \in \operatorname{SSS}(x)$ for any given $x \in B_{n}$ in normal form of canonical length $\ell(x)=\ell$. The algorithm can even output an explicit conjugating element between $x$ and $y$.

For the rest of the paper, we shall mostly be dealing with braids which lie in their own Super Summit Set (because pushing braids into their own SSS only costs $O\left(\ell^{2}\right)$, as we have just seen).

\section{Proofs of Theorems 1.1 and $\mathbf{1 . 3}$}

Throughout the section we shall assume Theorem 1.2 holds. Provided with the precise definitions and vocabulary related to the conjugacy problem in Garside groups, we now proceed to prove Theorems 1.3 and 1.1. The plan is to prove Theorem 1.3 first, and then to prove the validity of the algorithm described in the Introduction and to analyse its complexity.

First we recall one of the main results of [2]. 
Theorem 3.1 ([2, Theorem 3.37]). Let $x \in B_{n}$ be a pseudo-Anosov braid. Then there exists an integer $m$ such that $x^{m}$ is conjugate to a rigid braid. Moreover, the integer $m$ can be bounded independently of the length of $x$, namely, $m<\left(\frac{n(n-1)}{2}\right)^{3}$ for the classical Garside structure and $m<(n-1)^{3}$ for the dual structure.

The second main ingredient in Theorem 1.3 is Masur-Minsky's linear conjugacy bound [23], through the following result from [8] whose proof relies on the latter bound. (Recall that $\mathfrak{s}$ denotes the cyclic sliding operation - see Definition 2.5.)

Proposition 3.2 ([8, Theorem 2]). There exists a constant $C$, depending only on $n$ and on the chosen Garside structure, with the following property: if $x \in B_{n}$ is a pseudo-Anosov braid lying in its own Super Summit Set, and if $x$ possesses some rigid conjugate, then the conjugate $\mathfrak{s}^{C|x|}(x)$ is rigid.

This proposition yields a quadratic time algorithm for finding a rigid conjugate $y$ of any given pseudo-Anosov braid $x$ satisfying $x \in \operatorname{SSS}(x)$, and also for finding a conjugating element, provided a rigid conjugate exists at all.

We are now ready to prove Theorem 1.3.

Proof of Theorem 1.3. First recall that $n$ is considered fixed. Let us denote $\beta(n)$ the upper bound on $m$ in the statement of Theorem 3.1. Let $x, y \in B_{n}$ be pseudoAnosov braids. Our aim is to algorithmically find rigid conjugates of $x^{s}$ and $y^{s}$ for some $s \in \mathbb{N}$.

Due to Theorem 3.1, there exist two integers $i_{x}$ and $i_{y}$, both smaller than $\beta(n)$, such that $x^{i_{x}}$ and $y^{i_{y}}$ are conjugate to rigid braids. For all $i=1, \ldots, \beta(n)-1$ simultaneously, our algorithm iterates the operation $s$ starting from $x^{i}$, until a rigid braid is found. The corresponding power $i_{x}$, and a braid $z_{x}$ such that $\left(x^{i_{x}}\right)^{z_{x}}$ is rigid, are memorized. We denote $\tilde{x}$ this rigid conjugate of $x^{i_{x}}$. The same procedure, applied to $y$, yields an integer $i_{y}$, and braids $z_{y}$ and $\tilde{y}$ with the corresponding properties.

Note that the algorithm so far is doable in time $O\left(\ell^{2}\right)$, where $\ell$ is the maximum of the canonical lengths of $x$ and $y$. In order to prove this, we remark that the canonical length of all the braids $x^{i}$ and $y^{i}$, for $i=1, \ldots, \beta(n)-1$, is bounded above by $\beta(n) \ell$. By Theorem 2.9 and Proposition 3.2, the number of iterations needed in order to find $\tilde{x}$ is linearly bounded with respect to this length $\beta(n) \ell$. Finally, each iteration of the operation $\mathfrak{s}$ on a braid of canonical length $\ell$ takes time $O(\ell)$ (see [20]).

Let $s=\operatorname{lcm}\left(i_{x}, i_{y}\right)$. Since powers of rigid braids are again rigid, $x^{s}$ and $y^{s}$ are conjugate to rigid braids. So all our algorithm has to do now is to calculate $s$, and 
output

$$
\bar{x}=\tilde{x}^{\frac{s}{i_{x}}}, \quad \bar{y}=\tilde{y}^{\frac{s}{i_{y}}} \quad \text { and } \quad z_{1}=z_{x}, \quad z_{2}=z_{y} .
$$

This satisfies the requirements of Theorem 1.3.

Proof of Theorem 1.1. We have to prove that the algorithm described in the introduction is valid and of complexity $O\left(\ell(x)^{3}\right)$. Steps (1) and (3) are both of complexity $O\left(\ell^{2}\right)$, as was shown in [9], while Step (2) is of complexity $O\left(\ell^{3}\right)$ (see [4, Theorem 1]). Step (4) is of complexity $O\left(\ell^{2}\right)$, by Theorem 1.3. Finally, [20, Theorem 4.11] ensures us that [20, Algorithm 3] correctly solves CDP and CSP for rigid braids of length at most $\ell$ in time $O(\ell \cdot \kappa)$, where $\kappa$ denotes the cardinality of the sets of Sliding Circuits of the input braids. Our Theorem 1.2 now implies that Step (5) of our algorithm has complexity $O\left(\ell^{3}\right)$. Finally, Step (5) gives the correct answer, because of [21, Section 4.2]. Indeed, it is shown there that, for any $m \in \mathbb{N}$, any pseudo-Anosov braid has at most one $m$ th root, so that the relation

$$
\bar{x}=\bar{y}^{c}
$$

for a braid $c$ (i.e. $\left.\left(x^{s}\right)^{z_{1}}=\left(\left(y^{s}\right)^{z_{2}}\right)^{c}\right)$ is equivalent to the relation

$$
x^{z_{1}}=y^{z_{2} c},
$$

which is in turn equivalent to $x$ being conjugate to $y$ by $z_{2} c z_{1}^{-1}$.

It therefore remains to prove Theorem 1.2; this occupies the rest of the paper.

\section{Prerequisites for the proof of Theorem $\mathbf{1 . 2}$}

We advise the reader that none of the results in this section are new; however, we shall introduce some non-standard notation which will be useful in the proof.

\subsection{Sets of Sliding Circuits of rigid elements}

Our aim is to describe the structure of the set of Sliding Circuits of a rigid element of a Garside group. We use the same notations as in Section 2. The following results are proven in [19], for any element rigid or not. First we note that the set $\operatorname{SC}(x)$ is stable under conjugation by $\Delta$, cycling and decycling.

Definition 4.1 ([19]). Let $x \in G$ and $y \in \mathrm{SC}(x)$. A simple, non-trivial element $s$ of $G$ is said to be a minimal arrow for $y$ if $y^{s} \in \mathrm{SC}(x)$ and if the only positive prefixes $t$ of $s$ with $y^{t} \in \mathrm{SC}(x)$ are $t=1$ and $t=s$.

Proposition 4.2 ([19], see also [3, Corollary 2.7]). Let $x \in$ G. For every $y \in \operatorname{SC}(x)$, the minimal arrows for $y$ are prefixes of $\iota(y)$ or of $\partial(\varphi(y))$. 
Definition 4.3 ([19]). To every element $x$ of $G$ we associate a connected, oriented graph $\operatorname{SCG}(x)$ describing the set $\mathrm{SC}(x)$ as follows:

- the graph has one vertex for every element of $\operatorname{SC}(x)$,

- for every element $y$ of $\operatorname{SC}(x)$ and every minimal arrow $s$ for $y$, the graph $\operatorname{SCG}(x)$ has an oriented edge from the vertex $y$ to the vertex $y^{s}$. This edge is labelled $s$.

When $x$ has a rigid conjugate, the graph $\operatorname{SCG}(x)$ has a particularly elegant structure, which we describe now. Our study is based mainly on the following proposition from [19]:

Proposition 4.4 ([19, Theorem 1]). Let $x \in G$. Suppose that $x$ has a rigid conjugate. Then $\mathrm{SC}(x)$ is precisely the set of all rigid conjugates of $x$.

Definition 4.5. Let $x \in G$ be a rigid element, and let $y \in \operatorname{SC}(x)$. The orbit of $y$ is the set $O_{y}=\left\{\tau^{k} \mathbf{c}^{l}(y): k, l \in \mathbb{N}\right\}$.

Lemma 4.6. Let $x \in G$ be a rigid element, and let $y \in \operatorname{SC}(x)$.

(i) The orbit $O_{y}$ is a subset of $\mathrm{SC}(x)$.

(ii) The orbit $O_{y}$ is stable under cycling, decycling, and $\tau$; in particular, for every $z \in O_{y}, z^{\iota(z)}$ and $z^{\partial(\varphi(z))}$ are elements of $O_{y}$.

(iii) Let $y_{1}, y_{2} \in \operatorname{SC}(x)$. Then $O_{y_{1}} \neq O_{y_{2}}$ if and only if $O_{y_{1}} \cap O_{y_{2}}=\emptyset$.

(iv) The cardinality of the orbit $O_{y}$ is bounded above by $f \cdot \ell(y)$, where $f$ is the order of $\tau$.

Proof. Just observe that cycling and decycling induce cyclic permutations (up to $\tau$ ) of the non- $\Delta$ factors of the normal form of a rigid element.

For the rest of this subsection we shall always suppose that $x$ is rigid. The relation $\sim$, defined by $x \sim y$ if and only if $O_{x}=O_{y}$, is an equivalence relation on $\operatorname{SC}(x)$, and $\operatorname{SC}(x)$ is the disjoint union of the different orbits $O_{y}$. We denote by $\widetilde{\mathrm{SC}}(x)$ the quotient set $\mathrm{SC}(x) / \sim$. We now associate a "quotient graph" $\widetilde{\mathrm{SCG}}(x)$ to $\widetilde{\mathrm{SC}}(x)$ in the same way as $\operatorname{SCG}(x)$ is associated to $\operatorname{SC}(x)$. In order to do this rigorously, we need the following definition:

Definition 4.7. Let $y \in \mathrm{SC}(x)$ be rigid, and let $s$ be a minimal arrow for $y$. We say $s$ is a minimal useful arrow if $y^{s} \notin O_{y}$.

Remark 4.8. According to Proposition 4.2 and Lemma 4.6 (ii), the minimal useful arrows for $y$ are strict prefixes of $\iota(y)$ or of $\partial(\varphi(y))$. 
We recall the notion, due to Gebhardt [18], of the transport under cycling of an arrow: if $y, s \in G$, we define the transport under cycling of $s$ at $y$ by the formula $s_{y}^{(1)}=\iota(y)^{-1} s \iota\left(y^{s}\right)$. It is known ([18, Corollary 2.7]) that the transport induces a bijection between the set of minimal arrows for $y \in \operatorname{SC}(x)$ and the set of minimal arrows for $\mathbf{c}(y)$. Similarly, conjugation by $\Delta$ induces a bijection between the minimal arrows for $y$ and the minimal arrows for $\tau(y)$. In particular, if $s$ is a minimal useful arrow between $y$ and $y^{s}$, then $s_{y}^{(1)}$ is a minimal useful arrow between $\mathbf{c}(y)$ and $\mathbf{c}\left(y^{s}\right)$, and $\tau(s)$ is a minimal useful arrow between $\tau(y)$ and $\tau\left(y^{s}\right)$. Thus we can define the desired quotient graph without any ambiguity (i.e. the arbitrary choices made in the following definition do not matter):

Definition 4.9. To every rigid element $x$ of $G$ we associate a connected, oriented graph $\widetilde{\mathrm{SCG}}(x)$ as follows:

- The vertices of the graph correspond to elements of $\widetilde{\mathrm{SC}}(x)$.

- For every element $O_{y} \in \widetilde{\mathrm{SC}}(x)$, we arbitrarily choose a representative $y^{\prime}$ of $O_{y}$. Now, to any minimal useful arrow $s$ for $y^{\prime}$, from $y^{\prime} \in O_{y}$ to $z^{\prime} \in O_{z}$, we associate an edge of the graph, oriented from $O_{y}$ to $O_{z}$.

In order to bound the size of $\operatorname{SC}(x)$, it suffices to bound the number of vertices of $\widetilde{\mathrm{SCG}}(x)$ : if $\widetilde{\mathrm{SCG}}(x)$ has $k$ vertices, then the cardinality of $\mathrm{SC}(x)$ is at most $k \cdot f \cdot \ell(x)$ (due to Lemma 4.6 (iv)).

\subsection{The dual structure of $B_{4}$}

A detailed account of the dual Garside structure on braid groups can be found in the original article [5], and an introduction in [14, Chapter VIII]. We restrict ourselves here to a brief description of this structure in the case of the four-strand braid group $B_{4}$. We consider the sub-monoid $\mathrm{BKL}_{4}^{+}$of $B_{4}$ generated by the braids $a_{p, q}$, $1 \leqslant p<q \leqslant 4$, where

$$
\begin{aligned}
a_{p, p+1} & =\sigma_{p} \text { for } p=1, \ldots, 3, \\
a_{1,3} & =\sigma_{2}^{-1} \sigma_{1} \sigma_{2}, \\
a_{2,4} & =\sigma_{3}^{-1} \sigma_{2} \sigma_{3}, \\
a_{1,4} & =\sigma_{3}^{-1} \sigma_{2}^{-1} \sigma_{1} \sigma_{2} \sigma_{3} .
\end{aligned}
$$

The notation BKL is derived from the names of the discoverers of this structure: Birman, Ko and Lee. The monoid $\mathrm{BKL}_{4}^{+}$induces a partial order relation on the group $B_{4}: x \preccurlyeq y$ if and only if $x^{-1} y \in \mathrm{BKL}_{4}^{+}$. Taking as Garside element the braid $\delta=\sigma_{1} \sigma_{2} \sigma_{3}$, these data give rise to a new Garside structure, which we 
denote by $\mathrm{BKL}_{4}$. For instance, we shall write $x \in \mathrm{BKL}_{4}$ in order to say that $x$ is a four-strand braid seen in the structure $\mathrm{BKL}_{4}$, and given as a product of the generators $a_{i, j}$.

We now introduce some notation concerning the $\mathrm{BKL}_{4}$-structure which we shall be using for the rest of the article. We recall that $B_{4}$ can be seen as the mapping class group of the four times punctured disk $\mathbb{D}_{4}$. In the context of the BKL $_{4}$-structure it is practical to parametrize $\mathbb{D}_{4}$ as the unit disk in $\mathbb{C}$ with punctures

$$
P_{j}=\frac{1}{2} e^{-\frac{i(2 j-1) \pi}{4}}, \quad j=1, \ldots, 4 .
$$

The braid $a_{p, q}$ then corresponds to the counterclockwise half Dehn-twist along the arc $\left(P_{p}, P_{q}\right)$. Pictorially, we will represent the braid $a_{p, q}$ by the segment $\left(P_{p}, P_{q}\right)$; for instance, $a_{2,4}$ is denoted by $(\because), a_{1,4}$ is written $(: 1)$, and so on. Similarly, the braid which cyclically exchanges $P_{3}, P_{2}$ and $P_{1}$ by a counterclockwise movement is denoted $(\triangle)$. With this notation, the generators $a_{p, q}$ are subject to the following relations:

$$
\begin{array}{ll}
(\because)(!)=(!)(\because)=(!), & (\because)(\because)=(\because)(\because)=(\because), \\
(\because)(!)=(!)(\therefore)=(\therefore)(\because)=(\triangle), & (\because)(\because)=(\because)(\because)=(\because)(!)=(\vee), \\
(\because)(\because)=(\because)(\because)=(\therefore)(\because)=(\triangle), & (\because)(\because)=(\because)(\because)=(\because)(\because)=(\because) .
\end{array}
$$

The Garside element is $\delta=(\square)$. Conjugation by $\delta$ corresponds to a one-quarter counterclockwise turn, and $\tau$ is an automorphism of order 4 of $B_{4}$. Therefore Lemma 4.6 (iv), applied to the $\mathrm{BKL}_{4}$-structure, states that the orbit of a rigid braid $x$ contains at most $4 \cdot \ell(x)$ elements.

Our proof of Theorem 1.2 is based on the simplicity of the lattice of simple elements of $\mathrm{BKL}_{4}$. It has only fourteen elements:

$$
1,(\because),(!),(\because),(\because),(\vdots),(\because),(\triangle),(\nabla),(\triangleright),(\because),(!),(\because), \delta .
$$

The relations listed above are length-preserving (the word length on the letters $\left.a_{p, q}\right)$. This allows us to define a morphism $\lambda: B_{4} \rightarrow \mathbb{Z}$ by sending every braid $a_{p, q}$ to 1 . For any braid $x$, we call $\lambda(x)$ the weight of $x$. We have $\lambda(\delta)=3$ and, for any other simple non-trivial element $s$, we have $\lambda(s)=1$ or 2 . This observation (as already noted in [6]) yields a new quantity, in addition to canonical length, supremum and infimum, which is constant inside the Super Summit Set:

Lemma 4.10. Let $x \in \mathrm{BKL}_{4}$, and let $y \in \operatorname{SSS}(x)$. For every $z \in \operatorname{SSS}(x)$, the normal form of $z$ contains as many factors of weight 2 and as many factors of weight 1 as the normal form of $y$. 
Proof. For every braid $x \in \mathrm{BKL}_{4}$, if $k_{1}$ is the number of factors of weight 1 and $k_{2}$ the number of factors of weight 2 in the normal form of $x$, then $\ell(x)=k_{1}+k_{2}$ and $\lambda(x)=3 \inf (x)+2 k_{2}+k_{1}$. Thus $k_{1}$ and $k_{2}$ are constant in the Super Summit Set, since canonical length, weight, and infimum are constant there.

Again concerning the weight of the factors of a normal form, we make the following simple observation which will be needed later.

Remark 4.11. Recall that if $x$ has normal form $\delta^{p} x_{1} \cdots x_{r}$, then its inverse $x^{-1}$ has normal form $\delta^{-p-r} x_{r}^{\prime} \ldots x_{1}^{\prime}$, where $x_{i}^{\prime}=\tau^{-p-i}\left(\partial\left(x_{i}\right)\right)$ ([2, Theorem 1.5] for any Garside group). In particular, we have for the $\mathrm{BKL}_{4}$-case $\lambda\left(x_{i}^{\prime}\right)=3-\lambda\left(x_{i}\right)$.

The following very simple remark will turn out to be very useful:

Remark 4.12. Let $a$ and $b$ be two simple elements for BKL 4 . If $a$ is of weight 2 and $\delta$ does not divide the product $a b$, then the pair $a \cdot b$ is left-weighted.

We finally claim that in the $\mathrm{BKL}_{4}$-structure, the existence of a minimal useful arrow $s$ from $y^{\prime} \in O_{y}$ to $z^{\prime} \in O_{z}$ is equivalent to the existence of a minimal useful arrow from $z^{\prime} \in O_{z}$ to some element of $O_{y}$. (In other words, every edge in $\widetilde{\mathrm{SCG}}(x)$, for $G=\mathrm{BKL}_{4}$, is oriented both ways.) Let us prove this claim. According to Remark 4.8, such a minimal arrow $s$ is a strict prefix either of $\iota\left(y^{\prime}\right)$ or of $\partial\left(\varphi\left(y^{\prime}\right)\right)$. In particular, $\lambda(s)=1$. Now there is an arrow, which is of weight 1 and thus minimal, given in the first case by $s^{-1} \iota\left(y^{\prime}\right)$ from $z^{\prime}$ to $\mathbf{c}\left(y^{\prime}\right)$, and in the second case by $s^{-1} \partial\left(\varphi\left(y^{\prime}\right)\right)$, from $z^{\prime}$ to $\tau \mathbf{d}\left(y^{\prime}\right)$.

\section{Proof of Theorem 1.2}

Throughout this section we use the dual Garside structure on $B_{4}$. Our aim is to prove Theorem 1.2, so we consider a rigid pseudo-Anosov braid $x$, and we try to bound the size of $\operatorname{SC}(x)$. The hypothesis that $x$ is pseudo-Anosov implies that the canonical length of $x$ is strictly larger than 1 (this can be proven by analysing all braids with canonical length 1 ).

We shall see that it suffices to prove Theorem 1.2 separately in three special cases, which are defined in terms of the simple factors occurring in $x$ (see Section 4.2). We will consider successively the following three cases:

- The normal form of $x$ contains at least one factor of weight 1 and one factor of weight 2 - this case is solved in Proposition 5.1. (Notice that all other elements of $\operatorname{SC}(x)$ will have the same property, by Lemma 4.10.) 
- There exists an element $y$ of $\operatorname{SC}(x)$ such that all the factors other than $\delta$ occurring in the normal form of $y$ belong to $\{(1:),(\because),(\because),(\because)\}-$ this case is solved in Proposition 5.3.

- For every element $y$ of $\operatorname{SC}(x)$, all the factors other than $\delta$ occurring in the normal form of $y$ are of weight 1 , and at least one of them is $(\therefore)$ or $(\because)-$ this case is solved in Proposition 5.12.

In the first two cases, the hypothesis that $x$ should be pseudo-Anosov is in fact unnecessary. In these cases, we even construct a linear bound on \#SC $(x)$. The third case requires much more sophisticated techniques, and gives rise to an example showing that the quadratic bound is optimal.

\subsection{A simple special case}

We now describe a simple special case where Theorem 1.2 can be proved by elementary arguments.

Proposition 5.1. Let $x \in \mathrm{BKL}_{4}$ be a rigid braid whose normal form contains at least one factor of weight 1 and at least one factor of weight 2. Then the set $\mathrm{SC}(x)$ consists only of $O_{x}$ and in particular $\# \operatorname{SC}(x) \leqslant 4 \cdot \ell(x)$.

Proof. We shall see that no strict prefix of $\iota(x)$ (nor of $\partial(\varphi(x)))$ can conjugate $x$ to a rigid braid and in particular $x$ has no minimal useful arrow. Suppose on the contrary that $t \prec \iota(x)$ and that $x^{t}$ is rigid. Then, using the fact that the transport under cycling (already alluded to above in the paragraph before Definition 4.9) preserves the order $\preccurlyeq([18$, Corollary $2.2(\mathrm{~b})])$, we see that the transport $t^{(1)}$ of $t$ satisfies $1 \prec t^{(1)} \prec \iota(\mathbf{c}(x))$ (and conjugates $\mathbf{c}(x)$ to the rigid braid $\mathbf{c}\left(x^{t}\right)$ ). Iterating this argument we see that all factors of $x$ must have weight 2 , contradicting our hypothesis. On the other hand, if $t$ were a strict prefix of $\partial(\varphi(x))$, the same line of argument applied to $x^{-1}$ would finally establish that all factors of $x$ have weight 1 (Remark 4.11). The latter part of the claim in Proposition 5.1 follows immediately from the former together with Lemma 4.6 (iv).

Now, in order to prove Theorem 1.2, we have to find a quadratic bound on the size of $\mathrm{SC}(x)$ for any rigid pseudo-Anosov braid $x \in \mathrm{BKL}_{4}$. By Proposition 5.1, we can restrict our attention to braids whose normal form has all its factors (other than $\delta$ ) of the same weight ( 1 or 2 ). Up to considering inverses, we can restrict ourselves to the case of weight 1 (see [3, Section 3.1], where it is shown that when $x$ is rigid (and so is $x^{-1}$ ), the graphs $\operatorname{SCG}(x)$ and $\operatorname{SCG}\left(x^{-1}\right)$ are isomorphic).

So, for the rest of the proof of Theorem 1.2, we can suppose that $x$ is a rigid pseudo-Anosov braid whose normal form has only factors of weight 1 (i.e. ( $\because$ ), 
$(1:),(\because),(: 1),(\therefore),(\because))$, and $\delta^{ \pm 1}$. By Lemma 4.10 , all elements of $\operatorname{SSS}(x)$ have the same property. Moreover, using Remark 4.8, we see that for every $y \in \operatorname{SC}(x)$, all possible minimal useful arrows for $y$ are strict prefixes of $\partial(\varphi(y))$ (there is no strict non-trivial prefix of $\iota(y)$ because $\lambda(\iota(y))=1)$. In particular, all vertices of $\widetilde{\mathrm{SCG}}(x)$ have degree at most 3 .

We make one more simple, but very useful general observation:

Lemma 5.2. Suppose that the normal form of the rigid braid $y \in \operatorname{SC}(x)$ has only factors of weight 1 (and $\left.\delta^{ \pm 1}\right)$, with at least one factor equal to $(\because)$ or to $(\therefore)$. Then the vertex $O_{y}$ of $\widetilde{\mathrm{SCG}}(x)$ is of degree at most 2.

Proof. Up to replacing $y$ by $y^{\prime} \in O_{y}$, we can suppose that $\varphi(y)=(\therefore)$. But

$$
\partial((\therefore))=(\Xi),
$$

and this simple element has only two strict positive prefixes.

We split the rest of our argument into two parts. In Section 5.2, we study the case where $\operatorname{SC}(x)$ contains an element that does not satisfy the hypotheses of Lemma 5.2, i.e. an element whose normal form contains, apart from $\delta^{ \pm 1}$, only the letters from $\{(\because),(!:),(\because),(: !)\}$; we shall denote the latter set by $\mathbb{E}$. By contrast, Section 5.3 deals with the case where all elements of $\operatorname{SC}(x)$ satisfy the hypotheses of Lemma 5.2.

\subsection{Some element of $\operatorname{SC}(x)$ has all its factors in $\mathcal{E}$}

We recall the notation $\mathcal{E}=\{(\mathrm{l}:),(\because),(: \mathrm{l}),(\because)\}$. Our aim in this subsection is to prove the following result, whose proof is elementary but involves a lot of careful case-checking:

Proposition 5.3. Let $x \in \mathrm{BKL}_{4}$ be a rigid braid. Let us suppose that $\mathrm{SC}(x)$ contains some element $y$ whose normal form has all of its factors (apart from $\delta^{ \pm 1}$ ) belonging to $\mathcal{E}$. Then the graph $\widetilde{\mathrm{SCG}}(x)$ has at most six vertices. Moreover,

$$
\# \mathrm{SC}(x) \leqslant 24 \cdot \ell(x)
$$

The last sentence of Proposition 5.3 follows immediately from the preceding one, together with Lemma 4.6 (iv).

First we note that, in order to prove Proposition 5.3, it suffices to prove that, for some non-zero integer $m \in \mathbb{N}$, the graph $\widetilde{\mathrm{SCG}}\left(x^{m}\right)$ has at most six vertices. Indeed, since all braids in $\mathrm{SC}(x)$ are rigid, there is an injection from $\widetilde{\mathrm{SC}}(x)$ to $\widetilde{\mathrm{SC}}\left(x^{m}\right)$, sending an orbit $O_{y}$ to an orbit $O_{y^{m}}$. 
So possibly after replacing $x$ by $x^{4}$, we can suppose that $\inf (x)$ is a multiple of 4 . In fact, since for any integer $m$, multiplication by $\delta^{4 m}$ induces an isomorphism between $\operatorname{SC}(x)$ and $\operatorname{SC}\left(\delta^{4 m} x\right)$, we can even suppose that $\inf (x)=0$ (and thus that the infimum of any element of $\operatorname{SSS}(x)$ is zero).

So for the rest of the proof of Proposition 5.3, we shall assume that for $y$ (and hence for all elements of $O_{y}$ ) the normal form has all letters belonging to $\&$.

Remark 5.4. Conjugation by $\delta$ induces a permutation of $\mathcal{E}$. Moreover, for all $s, t \in \mathcal{E}$, the product $s t$ is in normal form if and only if $t \in\{s, \tau(s)\}$.

Remark 5.4 allows us to give a precise description of the normal form of $y$ :

Lemma 5.5. Let $y \in \mathrm{BKL}_{4}$ be a rigid braid with $\inf (y)=0$, all of whose factors belong to $\&$. Then, possibly after replacing $y$ by another element of $O_{y}$, the normal form of $y$ is of the form

$$
y=\prod_{j=1}^{r} \tau^{-r+j}\left((!:)^{k_{j}}\right),
$$

where $k_{j}, j=1, \ldots, r$, are strictly positive integers, and $r=1$ or $r \equiv 0(\bmod 4)$.

Proof. Up to conjugating $y$ by a power of $\delta$, we can suppose that $\varphi(y)=(1:)$. By Remark 5.4 and our hypothesis on $y$, the normal form of $y$ is indeed a product of the form

$$
y=\tau^{-(r-1)}\left((\mathrm{l}:)^{k_{1}}\right) \cdots(\mathrm{l}:)^{k_{r}}
$$

for integers $r$ and $k_{1}, \ldots, k_{r}$ all strictly positive. Then, due to rigidity and Remark 5.4, we have $\iota(y)=\varphi(y)$ or $\iota(y)=\tau(\varphi(y))$. Let us suppose that $r>1$. Up to cycling, we can suppose $\iota(y)=\tau(\varphi(y))$, which means that

$$
\tau^{-r+1}((!:))=\tau((!:)) .
$$

This implies that $r \equiv 0(\bmod 4)$.

Lemma 5.6. If $r=1$ in Lemma 5.5, then $\# \operatorname{SC}(x)=6$.

Proof. If $r=1$, then $\operatorname{SC}(x)=\left\{(!:)^{k_{1}},(\because)^{k_{1}},(\because !)^{k_{1}},(\because)^{k_{1}},(\because)^{k_{1}},(\because)^{k_{1}}\right\}$.

Lemma 5.7. Suppose that $r>1$ in Lemma 5.5. Then there exists a minimal arrow for $y$ if and only if $r \equiv 0(\bmod 3)$. If this is the case, then $y$ admits in fact three minimal (but not necessarily useful) arrows. If not, then the graph $\widetilde{\mathrm{SCG}}(x)$ has a single vertex. 
Proof. According to Lemma 5.5, we have $r \equiv 0(\bmod 4)$, and we can rewrite

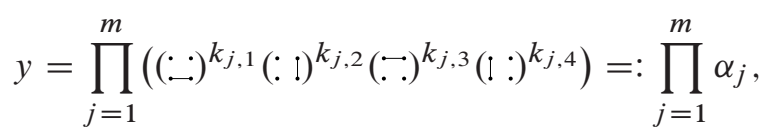

with $m:=\frac{r}{4}$ and $k_{j, i}>0$ for all $j, i$ with $1 \leqslant j \leqslant m$ and $1 \leqslant i \leqslant 4$. The minimal useful arrows for $y$, if they exist, are all strict prefixes of $\partial((1:))$, so they are $(\because),(: !)$ or $(\therefore)$.

The proof of the lemma essentially comes down to the following calculations, where the right hand sides of the equations (except for their first factor) are always in normal form; in other words, $A_{j}, B_{j}$ and $C_{j}$ are normal forms, independently of the powers occurring in the formulae (this calculation uses the notation $\alpha_{j}$ defined in the previous paragraph):

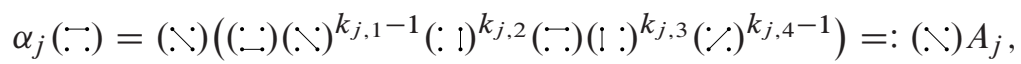

$$
\begin{aligned}
& \alpha_{j}(\therefore)=(: \mathrm{l})\left((\because)(\because \mathrm{l})^{k_{j, 1}}(\because)^{k_{j, 2}-1}(\because)^{k_{j, 3}}(\mathrm{l}:)(\because)^{k_{j, 4}-1}\right)=:(: !) B_{j} \text {, } \\
& \alpha_{j}(\because !)=(\because)\left((\because)^{k_{j, 1}}(: !)(\because)^{k_{j, 2}}(\vdots)^{k_{j, 3}-1}(\mathrm{l}:)^{k_{j, 4}}\right)=:(\because) C_{j} .
\end{aligned}
$$

We remark that, independently of $j$ and of the powers occurring, the "pairs" $A \cdot C, B \cdot A$ and $C \cdot B$ are in normal form. On the one hand, if $r \equiv 1$ or $r \equiv 2$ $(\bmod 3)$, then this shows that for every $u$ with $u \prec \partial((1:))$ we have $u \nprec y u$, and in particular $y^{u} \notin \operatorname{SSS}(x)$. On the other hand, if $m \equiv 0(\bmod 3)$, then this shows that the three braids

$$
\begin{aligned}
& y^{(\because)}=\left(\prod_{j=1}^{\frac{m}{3}} \alpha_{3 j-2} \alpha_{3 j-1} \alpha_{3 j}\right)^{(\bullet)}=\prod_{j=1}^{\frac{m}{3}} C_{3 j-2} B_{3 j-1} A_{3 j}, \\
& y^{(:)}=\prod_{j=1}^{\frac{m}{3}} B_{3 j-2} A_{3 j-1} C_{3 j}, \\
& y^{(\therefore)}=\prod_{j=1}^{\frac{m}{3}} A_{3 j-2} C_{3 j-1} B_{3 j}
\end{aligned}
$$

are rigid.

We suppose from now on that $r \equiv 0(\bmod 3)$ (this is always satisfied up to replacing $x$ by $x^{3}$ ). Let $u$ be a minimal useful arrow for $y$ (so implicitly we suppose that $\mathrm{SC}(x)$ is not reduced to the single orbit $O_{y}$ ). It follows in particular from our proof of Lemma 5.7 that we can always find, up to cyclic permutation of the 
factors, an element $z$ of $O_{y} u$ of the form

$$
z=\prod_{j=1}^{\frac{m}{3}} C_{3 j-2} B_{3 j-1} A_{3 j}
$$

by making an appropriate choice of indices and powers inside the factors.

We can then rewrite $y$ in the form

$$
y=\prod_{\nu=1}^{\frac{m}{3}}(\because)^{a_{v}}(\because !)^{b_{v}}(\because)^{c_{v}}(!:)^{d_{v}}(\because)^{e_{v}}(\because !)^{f_{v}}(\because)^{g_{v}}(!:)^{h_{v}}(\because)^{i_{v}}(\because !)^{j_{v}}(\because)^{k_{v}}(!:)^{l_{v}}
$$

with strictly positive integers $a_{v}, b_{v}, \ldots, l_{v}$ for all $v=1, \ldots, \frac{m}{3}$, and then $z$ becomes

$$
\begin{gathered}
z=\prod_{v=1}^{\frac{m}{3}}\left[(\because)^{a_{v}}(\because !)(\because)^{b_{v}}(\vdots)^{c_{v}-1}(!)^{d_{v}}(\because)(\because !)^{e_{v}}(\because)^{f_{v}-1}(\because)^{g_{v}}\right. \\
\left.(!:)(\because)^{h_{v}}(\vdots)^{i_{v}-1}(\because !)^{j_{v}}(\because)(!)^{k_{v}}(\because)^{l_{v}-1}\right] .
\end{gathered}
$$

Lemma 5.8. If the normal form of $z$ contains a factor $(\therefore)$ or $(\because)$, then $z$ admits a unique minimal useful arrow, i.e. the vertex $O_{z}$ of the graph $\widetilde{\operatorname{SCG}}(x)$ is a leaf.

Proof. Up to cycling or conjugating by $\delta$, we can suppose that the last factor of $z$ is $(\because)$, and that $l \frac{m}{3}>1$. There are at most two minimal arrows for $z$, namely (: $\left.:\right)$ and (!:). A calculation of the normal form of $z(1:)$ shows that (1:) $\nprec z(1:)$, which implies that $z^{(!:)} \notin \operatorname{SSS}(x)$, and hence the lemma.

In order to perform this calculation, we make three observations. Firstly,

$$
(\because)^{l_{\nu}-1}(!:)=(\because)(\because)(\because)^{l_{\nu}-2} \text {. }
$$

Secondly, for arbitrary integers $a, b>0$,

$$
\left((\because)^{a}(\because)(\mathrm{l}:)^{b}\right)(\because)=(1:)\left((\because !)^{a}(\because)(\mathrm{I}:)(\because)^{b-1}\right)
$$

and the first factor on the right hand side is independent of the powers $a$ and $b$. Thirdly, the pair $(\therefore)(1:)$ is in normal form. Now the previous calculation can be pushed towards the left along the normal form of $z$, getting twisted by a conjugation by $\Delta$ at each step, until it hits, possibly, a factor $(\therefore)$ or $(\because)$, where it gets stuck.

This shows that the multiplication of $z$ by ( $:$ :) on the right can only modify the beginning of the normal form of $z$ if $(\because)^{l^{\frac{m}{3}}-1}$ is the only occurrence of $(\because)$ or $(\therefore)$ in $z$. Moreover, if this is the case, then the initial factor of $z(!)$ is $(\because)$ and $(1:)$ is not a prefix of the latter. On the other hand, by our hypothesis that $O_{z}$ is distinct from $O_{y}$, there is a minimal arrow from $z$ to some element of $O_{y}$. This completes the proof. 
Lemma 5.9. Suppose that the normal form of $z$ does not contain any factor $(\because)$ or $(\therefore)$. Then

(i) the three strict prefixes of $\partial(\varphi(z))$ are minimal arrows for $z$,

(ii) if $v$ is a minimal useful arrow for $z$ conjugating $z$ to another rigid braid whose normal form contains no factor $(\because)$ or $(\therefore)$, then $z^{v} \in O_{y}$ and $v=(: \mathrm{l})$.

Proof. According to our hypothesis, we can further rewrite the formulae from the proof of Lemma 5.7:

$$
y=\prod_{v=1}^{\frac{m}{3}}(\because)^{a_{v}}(: 1)^{b_{v}}(\because)(\mathrm{l}:)^{d_{v}}(\because)^{e_{v}}(: 1)(\because)^{g_{v}}(1:)^{h_{v}}(\because)(: 1)^{j_{v}}(\because)^{k_{v}}(\mathrm{l}:)
$$

and

$$
z=\prod_{v=1}^{\frac{m}{3}}(\because)^{a_{v}}(: !)(\because)^{b_{v}}(1:)^{d_{v}}(\because)(: !)^{e_{v}}(\because)^{g_{v}}(1:)(\because)^{h_{v}}(: !)^{j_{v}}(\because)(!:)^{k_{v}} .
$$

(i) According to Lemma 5.7, $z$ admits three minimal (not necessarily useful) arrows.

(ii) Let $v$ be a minimal useful arrow for $z$ such that $z^{v}$ contains no factor $(\therefore)$ or $(\because)$. First at least one such arrow exists, because of our hypothesis $O_{y} \neq O_{z}$. We know that $v \in\{(\because),(: !),(\therefore)\}$. Thus it is sufficient to prove that $v \neq(\because)$ and $v \neq(\therefore)$. We are going to apply the formulae from the proof of Lemma 5.7, now with $z$ playing the rôle previously played by $y$.

If $v=(\therefore)$, then the formulae from the proof of Lemma 5.7, together with the restriction that $z^{v}$ contains neither ( $\vdots$ ) nor $(\because)$, imply $b_{v}=e_{v}=h_{v}=k_{v}=1$.

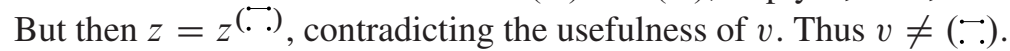

Analogously, if $v=(\therefore)$, then due to the formulae from the proof of Lemma 5.7 we obtain $a_{v}=d_{v}=g_{v}=j_{v}=1$. But then

$$
z=\prod_{v=1}^{\frac{m}{3}}(\because)(: !)(\because)^{b_{v}}(!:)(\because)(: !)^{e_{v}}(\because)(1:)(\because)^{h_{\nu}}(: !)(\because)(1:)^{k_{v}}
$$

and

$$
z(\therefore)=\prod_{v=1}^{\frac{m}{3}}(\because)(\because 1)(\because)(1:)^{b_{v}}(\because)(: 1)(\because)^{e_{v}}(1:)(\because)(: 1)^{h_{v}}(\because)(1:)(\because)^{k_{n u}-1} .
$$

We obtain $\mathbf{c}\left(z^{(\searrow)}\right)=\tau(z)$, contradicting the usefulness of $v$. Thus $v \neq(\therefore)$.

Lemma 5.9 shows that the graph $\widetilde{\mathrm{SCG}}(x)$ cannot contain a chain of three vertices whose elements contain no factor $(\because)$ or $(\because)$. By Lemma 5.8 any vertex 
which does contain at least one factor $(\therefore)$ or $(\because)$, but which is adjacent to a vertex which does not, is a leaf. Since the graph $\widehat{\operatorname{SCG}}(x)$ is connected and all vertices have degree at most 3 , this implies that it has at most six vertices. This completes the proof of Proposition 5.3.

\subsection{All elements of $\operatorname{SC}(x)$ have at least one factor not belonging to $\mathcal{E}$}

In this subsection we suppose that all elements of $\mathrm{SC}(x)$ have at least one factor in their normal form equal to $(\therefore)$ or $(\because)$. According to Lemma 5.2, the graph $\widetilde{\mathrm{SCG}}(x)$ is then a (possibly closed) line. In order to prove Theorem 1.2 , we need to bound the length of this line. This task seems much more difficult than in the previous subsections and we have currently no elementary proof of Theorem 1.2 under the above hypotheses. In order to illustrate the difficulty, we show first that the quadratic bound of Theorem 1.2 is optimal. The following example was obtained with the help of the program GAP [24]:

Example 5.10. For all $k \in \mathbb{N}$, the braid $\beta_{k}=(\because)(!)(\because)(\therefore)(\because)(\because)^{3 k}(\because)^{-3 k}$, whose normal form is

$$
\beta_{k}=(\because)(!:)(\because)(\because)(\because !)[(\because)(\because)(\because !)]^{k},
$$

is rigid and pseudo-Anosov with $\ell\left(\beta_{k}\right)=3 k+5$. Moreover, the graph $\widetilde{\operatorname{SCG}}\left(\beta_{k}\right)$ is a line with $3 k+2$ vertices. (Explicitly, in order to obtain braids representing all vertices of $\widetilde{\operatorname{SCG}}\left(\beta_{k}\right)$, it suffices to conjugate $\beta_{k}$ by $(\because)^{j}$, for $j=0, \ldots, 3 k+1$.) Thus \#SC $\left(\beta_{k}\right)=4 \cdot(3 k+2) \cdot(3 k+5)$.

Our proof of Theorem 1.2 under the hypotheses of this subsection resorts to Masur-Minsky's linear bound on the length of an element conjugating two pseudoAnosov elements of a mapping class group ([23, Theorem 7.2]).

We consider the length function $|\cdot|$ on $B_{4}$ induced by taking as generators of $B_{4}$ the set of divisors of $\delta$, i.e. the set of BKL-simple braids (see Remark 2.3). The result of Masur and Minsky, applied to the case of four-strand braids, then reads:

Theorem 5.11 ([8, Proposition 7]). There exists a constant $c$ such that for every pair $\left(z_{1}, z_{2}\right)$ of conjugate pseudo-Anosov four-strand braids, there exists a conjugating element $w\left(\right.$ i.e. $\left.z_{1}^{w}=z_{2}\right)$ such that $|w| \leqslant c \cdot\left(\left|z_{1}\right|+\left|z_{2}\right|\right)$.

We remark that the length function used in the statement of [8, Proposition 7] is the length associated to the alphabet of divisors of $\Delta$, i.e. the set of simple braids in the classical Garside structure. However, the length functions associated to different finite generating sets in a group are in bi-Lipschitz correspondence. 
More explicitly, our two length functions on $B_{4}$ are related, with the obvious notations, by the formula

$$
|x|_{\mathrm{BKL}_{4}} \leqslant 2 \cdot|x|_{\text {classical }} \leqslant 6 \cdot|x|_{\mathrm{BKL}_{4}} .
$$

In order to complete the proof of Theorem 1.2, it is now sufficient to prove the following result (where the constant $c$ is the one promised by Theorem 5.11).

Proposition 5.12. Let $x \in \mathrm{BKL}_{4}$ be a rigid pseudo-Anosov braid. Suppose that all elements of $\mathrm{SC}(x)$ have at least one factor of their normal form equal to $(. \vdots)$ or $(\because)$. Then the graph $\widetilde{\mathrm{SCG}}(x)$ has at most $16 \cdot c \cdot \ell(x)$ vertices. Thus,

$$
\# \mathrm{SC}(x) \leqslant 64 \cdot c \cdot \ell(x)^{2} .
$$

Proof. First we can suppose that $|x| \leqslant 2 \cdot \ell(x)$. In order to see this, we notice that multiplying $x$ by any power $m$ of the central element $\delta^{4}$ induces an isomorphism between the graphs $\widetilde{\operatorname{SCG}}(x)$ and $\widetilde{\mathrm{SCG}}\left(\delta^{4 m} x\right)$. In this way, we can suppose that $\inf (x) \in\{-3,-2,-1,0\}$. Then from Remark 2.3 we obtain $|x| \leqslant 2 \cdot \ell(x)$ (recalling that $\ell(x) \geqslant 2$, since $x$ is pseudo-Anosov).

According to Lemma 5.2, every vertex of the graph $\widetilde{\mathrm{SCG}}(x)$ has degree at most 2, so topologically the graph is either a compact line segment or a circle. We claim that any two distinct vertices $O_{a}, O_{b}$ in the graph $\widetilde{\mathrm{SCG}}(x)$ can be connected in the graph by a path of length at most $8 \cdot c \cdot \ell(x)$. Before proving this claim, we observe that the claim, together with Lemma 4.6 (iv), implies Proposition 5.12 (the factor 2 comes from the possibility that the graph might form a circle).

So let $O_{a}$ and $O_{b}$ be two distinct vertices of $\widetilde{\operatorname{SCG}}(x)$, and let $z_{a}$ and $z_{b}$ be representatives of these two orbits. Due to Theorem 5.11, there exists a braid $w$ satisfying $z_{a}^{w}=b_{b}$, and such that $|w| \leqslant 2 \cdot c \cdot|x| \leqslant 4 \cdot c \cdot \ell(x)$. Up to changing the representative $z_{a}$, we can suppose that $\inf (w)=0$. Then $\lambda(w) \leqslant 2 \cdot|w|$, as every factor of the normal form of $w$ contributes at most 2 to the weight of $w$. Thus $w$ is the product of at most $2 \cdot|w|$ minimal arrows, which yields a path of length at most $2 \cdot|w|$ between $O_{a}$ and $O_{b}$ in the graph $\widetilde{\operatorname{SCG}}(x)$.

Question 5.13. Open Question 2 in [2] concerns the existence of a polynomial bound in $n$ and $\ell$ on the size of the set of Sliding Circuits of a rigid (pseudoAnosov) braid with $n$ strands and of canonical length at most $\ell$. Prasolov gave a negative answer, by exhibiting a family of rigid pseudo-Anosov braids for which the size of the sets of Sliding Circuits grows exponentially as a function of $n$ (for both structures, dual and classical). On the other hand, if we fix $n$, then no such counter-example is known, and indeed in the special case $n=4$ our Theorem 1.2 gives an affirmative answer. So we formulate the following question: for any fixed 
integer $n$, does there exist a polynomial $P_{n}$ such that the cardinality of the (classical or dual) set of Sliding Circuits of a rigid pseudo-Anosov braid with $n$ strands is bounded above by $P_{n}(\ell(x))$ ?

Question 5.14. Is the size of the (classical or dual) Super Summit Set of a rigid pseudo-Anosov 4-braid $x$ bounded above by $P(\ell(x))$, for some polynomial $P$ ? We know from [10] that for braids with five or more strands, the size of the classical Super Summit Set can increase exponentially with the length of the braid.

Acknowledgments. The authors are grateful to Juan González-Meneses for suggesting a simplification in the proof of Theorem 1.2.

\section{Bibliography}

[1] J. Birman, Braids, Links and Mapping Class Groups, Ann. of Math. Stud. 82, American Mathematical Society, Providence, 1974.

[2] J. Birman, V. Gebhardt and J. González-Meneses, Conjugacy in Garside groups I: Cycling, powers and rigidity, Groups Geom. Dyn. 1 (2007), no. 3, 221-279.

[3] J. Birman, V. Gebhardt and J. González-Meneses, Conjugacy in Garside groups II: Structure of the ultra summit set, Groups Geom. Dyn. 2 (2008), no. 1, 16-31.

[4] J. Birman, V. Gebhardt and J. González-Meneses, Conjugacy in Garside groups III: Periodic braids, J. Algebra 316 (2007), no. 2, 746-776.

[5] J. Birman, K.-H. Ko and S.-J. Lee, A new approach to the word and conjugacy problems in the braid groups, Adv. Math. 139 (1998), no. 2, 322-353.

[6] J. Birman, K.-H. Ko and S.-J. Lee, The infimum, supremum and geodesic length of a braid conjugacy class, Adv. Math. 164 (2001), 41-56.

[7] E. Brieskorn and K. Saito, Artin-Gruppen und Coxeter-Gruppen, Invent. Math. 17 (1972), 245-271.

[8] M. Calvez, Fast Nielsen-Thurston classification of braids, Algebr. Geom. Topol., to appear.

[9] M. Calvez and B. Wiest, Fast algorithmic Nielsen-Thurston classification of fourstrand braids, J. Knot Theory Ramifications 21 (2012), no. 5, 1250043.

[10] S. Caruso, A family of pseudo-Anosov braids whose super-summit sets grow exponentially, J. Knot Theory Ramifications 22 (2013), no. 9, 1350050.

[11] A. Casson and S. Bleiler, Automorphisms of Surfaces after Nielsen and Thurston, London Math. Soc. Stud. Texts 9, Cambridge University Press, Cambridge, 1988.

[12] R. Charney and J. Meier, The language of geodesics for Garside groups, Math. Z. 248 (2004), no. 3, 495-509. 
[13] P. Dehornoy, Groupes de Garside, Ann. Sci. Éc. Norm. Supér. (4) 35 (2002), no. 2, 267-306.

[14] P. Dehornoy, I. Dynnikov, D. Rolfsen and B. Wiest, Ordering Braids, American Mathematical Society, Providence, 2008.

[15] E. Elrifai and H. Morton, Algorithms for positive braids, Q. J. Math. Oxford Ser. 2 45 (1994), no. 180, 479-497.

[16] A. Fathi, F. Laudenbach and V. Poenaru, Travaux de Thurston sur les surfaces. Séminaire Orsay, 2nd ed., Centre National de la Recherche Scientifique, Astérisque 6667, Société Mathématique de France, Paris, 1991.

[17] F. Garside, The braid groups and other groups, Q. J. Math. Oxford Ser. 220 (1969), 235-254.

[18] V. Gebhardt, A new approach to the conjugacy problem in Garside groups, J. Algebra 292 (2005), no. 1, 282-302.

[19] V. Gebhardt and J. González-Meneses, The cyclic sliding operation in Garside groups, Math. Z. 265 (2010), no. 1, 85-114.

[20] V. Gebhardt and J. González-Meneses, Solving the conjugacy problem in Garside groups by cyclic sliding, J. Symbolic Comput. 45 (2010), no. 6, 629-656.

[21] J. González-Meneses, The $n$th root of a braid is unique up to conjugacy, Algebr. Geom. Topol. 3 (2003), 1103-1118.

[22] S. J. Lee, Algorithmic solutions to decision problems in the braid groups, $\mathrm{PhD}$ thesis, Korea Advanced Institute of Science and Technology, 1999.

[23] H. Masur and Y. Minsky, Geometry of the complex of curves. II. Hierarchical structure, Geom. Funct. Anal. 10 (2000), no. 4, 902-974.

[24] The GAP group, GAP - Groups, Algorithms, and Programming, version 3, release 4, patchlevel 4, Lehrstuhl D für Mathematik, Rheinische Westfälische Technische Hochschule, Aachen, Germany, 1997, package CHEVIE.

Received August 1, 2013; revised March 13, 2014.

\section{Author information}

Matthieu Calvez, Departamento de Matemática y Ciencia de la Computación, Facultad de Ciencias, Universidad de Santiago de Chile, Avenida Libertador Bernardo O'Higgins 3363, Santiago, Chile.

E-mail: calvez.matthieu@gmail.com

Bert Wiest, IRMAR (UMR 6625 du CNRS), Université de Rennes 1, Campus de Beaulieu, 35042 Rennes Cedex, France.

E-mail: bertold.wiest@univ-rennes1.fr 